\title{
Distal Humerus External Rotation Osteotomy for Hand Position in Arthrogryposis
}

\author{
Lindley B. Wall, MD, Valeri Calhoun, MS, Summer Roberts, BS, and Charles A. Goldfarb, \\ MD \\ Department of Orthopaedic Surgery, Washington University School of Medicine, Shriners Hospital \\ for Children, St. Louis Missouri
}

\begin{abstract}
Purpose-In the amyoplasia type of arthrogryposis a reverse pronated grasp pattern is often seen. We hypothesized that repositioning the hands, through distal humerus external rotation osteotomies (DHO), would allow for palm-to-palm grasp without arm cross-over, and would improve function and parent/patient satisfaction.
\end{abstract}

Methods-The medical records of all patients treated surgically for arthrogryposis were reviewed at the Shriners Hospital in St. Louis, MO. From 2012-2014, nine patients (14 extremities) had undergone a DHO. All patients had pre-operative and post-operative video recordings of functional activities and we assessed functional changes after osteotomies. Preoperative upper extremity position was graded as $1=$ palms facing midline, $2=$ palm facing posterior, and 3=palms facing away from midline. Post-operative Pediatric Outcomes Data Collection Instrument (PODCI) questionnaires were obtained and parent satisfaction was evaluated.

Results-Mean patient age at the time of surgery was 6.5 years-old. There were 5 patients who underwent bilateral DHOs. All patients had 3 or fewer additional procedures on an upper extremity during the study period. All patients had an improved resting posture of the upper extremity after DHO surgery, with a mean change of 51 degrees (range 15-90 degrees). Grasp pattern was altered in 13 extremities; there was a change in hand position of at least one grade and 5 had complete change from 3 to 1 , palms facing away from midline to facing towards midline. There was a wide range in post-operative PODCI scores for function, but Happiness scores were high, mean 89 (range 60-100). Parents universally stated the procedure improved the child's function "a great deal". There were two complications, one periprosthetic humerus facture with recurrence of the internal rotation and one patient with scarring of the triceps requiring tenolysis.

Conclusions-The DHO is an effective procedure for correcting the internal rotation position of the upper extremity in arthrogryposis and the surgery improves hand opposition with minimal

Corresponding Author: Lindley B. Wall, M.D., Assistant Professor Orthopaedic Surgery, Washington University School of Medicine, 660 South Euclid Avenue, Campus Box 8233, St. Louis, MO 63110, 314-362-9369, wallli@wudosis.wustl.edu.

Publisher's Disclaimer: This is a PDF file of an unedited manuscript that has been accepted for publication. As a service to our customers we are providing this early version of the manuscript. The manuscript will undergo copyediting, typesetting, and review of the resulting proof before it is published in its final citable form. Please note that during the production process errors may be discovered which could affect the content, and all legal disclaimers that apply to the journal pertain. 
complications. Universally there was perceived improved function with high post-operative PODCI Happiness scores.

Level of Evidence-Therapeutic, Level V

\section{Keywords}

Arthrogryposis; Humerus Osteotomy; Hand Position; External Rotation Osteotomy

\section{Introduction}

Arthrogryposis is a condition characterized by joint contractures and underdevelopment of muscles. The term encompasses a large group of disorders $(1,2)$; amyoplasia is a nonsyndromic form of arthrogryposis that occurs sporadically with no known hereditary pattern. The characteristic upper extremity deformity includes shoulder internal rotation with limited motion, weak or absent elbow flexors with elbow extension contracture, flexed and ulnar deviated wrists with limited active extension, and minimal finger flexion and thumb-in-palm deformity (2-4).

Recent studies have reported outcomes of surgical treatment for the elbow and wrist contractures (1-10). While these surgeries improve function in the upper extremities, they do not change the grasp pattern of the hands. Dorsal closing wedge osteotomy of the carpus brings the wrists out of flexion; however, the palms may not be opposable if the shoulder internal rotation contractures are not addressed. The shoulder internal rotation typically positions the hands dorsal side to dorsal side (Figure 1), and necessitates that the arthrogrypotic patient use a crossover grasp, placing one hand over the other, to perform palm-to-palm grasp (Figure 2).

A single surgical technique paper has been published addressing the shoulder internal rotation deformity and repositioning of the extremity to improve bimanual activities including grasp (11). There have been no studies assessing the function and outcomes of these osteotomies in a series of patients. The current study reports on the outcomes of distal humerus external rotation osteotomy to improve hand position in arthrogryposis. We tested the null hypothesis that the distal humerus osteotomy (DHO) would not change the grasp pattern in patients with arthrogryposis, amyoplasia type.

\section{Methods}

After institutional review board approval was obtained, we prospectively enrolled patients with arthrogryposis, amyoplasia type, who were undergoing a distal humerus external rotation osteotomy to improve grasp. The investigation was performed at St. Louis Shriners Hospital. The decision to proceed with surgery was a result of a conversation between the family and the treating surgeon addressing the patient's grasp pattern. Indications for surgery included the absence of palm-to-palm grasp. A relative requirement prior to DHO is a neutral wrist, though the wrist osteotomy can be performed at the time of the DHO. If the patient underwent bilateral surgeries, both arms were included in the series although the surgeries were performed in a staged fashion. 
A certified hand therapist evaluated all patients. Before and at final follow-up, passive internal and external rotation of the shoulder and the resting posture of the extremity were measured. The resting posture was assessed when the patient was upright and the arm adducted to the side with the elbow flexed as close to 90 as possible; the resting posture is the measurement between the forearm and the body with the angle of 90 degrees from the body to the forearm as neutral. Video recordings were made of each patient while performing various grasping activities with different sized objects. These videos were taken preoperatively and postoperatively at 3 and 12 months. Two certified hand therapists then assessed the videos for grading of grasp patterns. Patients were graded based on grasp patterns, using an assessment system developed by the authors to describe hand position during grasp. Grade 1, palm facing midline; grade 2, palm facing posteriorly; and grade 3, palm facing away from midline. The parent and/or care-giver completed the Pediatric Outcomes Data Collection Instrument (PODCI) questionnaires preoperatively and postoperatively, to assess for overall health, pain, and ability to participate in activities $(12,13)$. Additionally, parents were asked for a subjective outcome assessment with a questionnaire allowing an open, free text response for their opinion of the surgery outcome, specifically in regards to the arm rotation from the DHO. A research coordinator who was not involved in the patients' care introduced this questionnaire.

\section{Surgical technique}

Passive shoulder rotation was based on clinical examination when the child was anesthetized; the rotation was assessed looking at the hand position with the extremity at the side of the body in shoulder adduction and the elbow in 90 degrees of flexion. If a posterior elbow release and triceps lengthening (9) was done concurrently, then it was performed first to allow assessment of the hand position with elbow flexion. The amount of external rotation correction was based on clinical examination with the goal to reposition the hand with the palm facing medially and to allow the hand to reach the mouth. We find this amount of rotation tends to be about 90 degrees, but each patient is independently assessed. No attempt was made to preserve positioning of the hand for perineal care.

All surgeries were performed with the patient under general anesthesia in the lateral decubitus position. If the upper arm was large enough, then a sterile tourniquet was used at a pressure of $250 \mathrm{mmHg}$. A posterior incision was made from the mid-aspect of the humerus to the olecranon. The ulnar nerve was identified and protected. The cutaneous branch of the radial nerve was also identified and dissected proximally to the radial nerve proper and both were protected throughout the case. The triceps was retracted and a transverse osteotomy was designed above the flare of the distal humeral metaphysis. Prior to osteotomy, a 2.4 or $2.7 \mathrm{~mm}$ 6-hole plate was placed along the posterior lateral column of the humerus and the proximal screw holes drilled. We completed the osteotomy with 0.045 Kirschner wire cortical perforation and osteotome separation of the fragments. The plate was then fixed proximally to the shaft and the distal fragment was secured once the distal fragment of the humerus was externally rotated. The skin was closed and the patient was placed into a longarm splint. 
Postoperatively the patient was seen in the office at 2 weeks and x-rays were obtained. Gentle elbow range of motion was begun and patients wore a sling for the following 6 weeks, except for bathing and exercises. Weight bearing was restricted until radiographic healing was seen, typically 2 months from surgery. The postoperative plan was modified based on concomitant surgeries. If there was a concomitant carpal wedge osteotomy, the wrist was continually immobilized for 6 weeks post-operatively, but elbow motion was allowed. When a DHO was performed at the same time as a posterior elbow release and triceps lengthening, motion was started at 2 weeks and a protective long arm splint, positioned with the elbow in 90 degrees of flexion, was utilized for 6 weeks. Radiographs of the humerus were followed until complete radiographic healing is achieved, assessed as 3 healed cortices, typically at approximately 3 months.

\section{Results}

Nine patients were included in the study, 3 were female. Five patients had bilateral surgeries so a total of 14 extremities were studied. Mean follow-up was 1.9 years (range 6 months to 4 years). The average age at the time of DHO surgery was 6.5 years (range 2-13 years). All patients had three or fewer additional procedures on a single extremity over the study period. There were 5 concurrent posterior elbow releases with triceps lengthening and 3 concurrent carpal wedge osteotomies. No patient had all three procedures performed at the same time. Other non-concurrent surgeries included thenar release, first web deepening, Steindler flexorplasty, and long head of the triceps transfer.

There were two complications. One patient sustained a humerus fracture adjacent to the plate after a fall and was treated successfully with sling immobilization. Upon healing there was recurrence of the internal rotation posture, thus this patient was not included in the data presented (Tables 1 and 2). A second patient required a revision posterior elbow release one year after a combined DHO and elbow release surgery because of stiffness and scarring resulting in loss of motion.

At one year after surgery, the average change of arm position was an increase of 51 degrees of external rotation (range, 15-90) measured in resting posture (Table 1). Assessing hand position by grade, there was typically a change in position by at least one grade of external rotation. (Table 2). However, five patients changed from grade 3 (palms facing away from midline) to 1 (hands facing toward midline). An example of such a change can be seen in the change in the pre-operative photo (Figure 2) compared to the post-operative follow up photo (Figure 3). This positioning was seen in the video recordings for each patient, though not all had a 2 level change in grade. The one patient who failed to improve on video assessment was the patient with the distal humerus fracture and recurrence of the internal rotation position.

Additionally, it was seen that handwriting improved in 5 patients. These 5 patients all had surgery on their dominant side and thus affected their writing. After DHO the hand holding the pen could rest with its ulnar border on the table, in contrast to the radial border or with the hand held above the paper with palm downward, which allowed improved posture for writing and holding the pen. 
While there was a wide range of post-operative PODCI scores for upper extremity function and global outcomes, the mean Happiness score revealed a high score of 89 (range 60-100) suggesting that patients were fairly happy. Parent responses were universally positive (Table 3). No negative responses were obtained on the written free text questionnaires.

\section{Discussion}

Limited joint motion and muscle development in patients with amyoplasia greatly impairs the ability to grasp objects and use the hands together. Given the typical limitations of finger motion, bimanual grasp and manipulation is highly advantageous but, in our opinion, is often limited by arm position (shoulder internal rotation). The internally rotated arm position typically prevents palm-to-palm grasping activities, the most effective position for such functions in this population. Multiple procedures have been described to improve the use of the upper extremities in these patients, such as dorsal carpal wedge osteotomy $(15,16)$, posterior elbow releases with triceps lengthening (9-10), and muscle transfers for active elbow flexion (1,5-8). While these procedures help a child to achieve hand to mouth contact, function remains limited if the hand remains externally rotated, pointing away from the midline. At our institution we have utilized a distal humerus osteotomy to reposition the hands for improved grasp. The patient with a cross over grasp or side-to-side grasp can have an improved bimanual grasp pattern by turning the palms toward midline after rotational osteotomy of the humerus. We have shown an average of 51 degrees rotational improvement in the clinical resting posture of the upper extremity. The change in clinical resting posture seen changed the hand position on average from Grade 3 (palms away from mid-line) to 2 (palms facing posterior) or a 2 to a grade 1 (palm to palm). In 5 extremities, we were able to change from 3 to grade 1 . We feel that these changes allowed for improvement in grasping.

We also found that the $\mathrm{DHO}$ changed the hand position to allow improved ease in writing and grasping of small objects in a majority of the patients. Our video assessment revealed that after DHO, patients could more easily pick up a one-inch cube with improved visualization, their palm not blocking their view, in addition to an improved grasp pattern. During pre-operative grasp, the patients were not able to see the object and it had to be placed into their hand for grasp because their palm facing away blocked their view (Video 1). After the osteotomy, we hypothesize that the improved visualization of the object also contributed to the patient's improved ability to grasp the object (Video 2). This new position also allowed the ulnar border of the hand to rest on the table, contributing to improvement in the ease of writing. While it was difficult to quantify the specific social and emotional effects of this procedure, in general, all patients were happy with their outcome.

The single report in the literature on this topic describes the surgical technique of the distal humerus osteotomy, performed concurrently with a posterior elbow release (11). The authors discuss rotating the distal arm through a DHO to allow the hand to reach the mouth, but not so much as to impair midline function; no specific degree of external rotation was recommended. We have come to incorporate approximately 90 degrees of rotation and have not yet had a case of excessive external rotation causing the patient to be unable to touch their hands to midline for grasp or to be unable to passively flex to the mouth (if their elbow allowed). In our earliest patients, our rotational corrections were limited but as we have 
become more comfortable with the functional improvement, we are more aggressive with rotational change and are rotating about 90 degrees intra-operatively. Additionally in the technique paper (11), the authors also describe utilizing cross Kirschner wires (K-wires) as an alternative option for osteotomy fixation but we no longer use K-wires. We feel that a plate gives more rigid fixation and allows for earlier motion in these children who already struggle with obtaining and maintaining motion. Lastly, our osteotomy location is similar to this previous surgical technique, the distal metaphysis of the humerus. We believe the distal humerus to be a very accessible surgical location and it allows the posterior elbow to be addressed simultaneously through the same incision.

Additionally, with regards to surgical assessment of rotation, we acknowledge that forearm pronation in some patients may contribute to the deformity of palms facing away from midline. We recommend that in patients with hyperpronation of the forearm, critical assessment of the effects of a DHO should be considered in order to prevent excessive external rotation with the elbow in flexion. Lastly, we have not had obvious recurrence of our rotation correction seen by loss of hand position at this short follow-up, except in one patient with the peri-implant fracture.

There was one post-operative fracture in our cohort. The patient fell after his osteotomy had healed and a fracture sustained just above the plate. This fracture was treated nonoperatively and healed with recurrent internal rotation. This case emphasized the risk of falls and possible fracture in this population. The arthrogrypotic patient is not able to easily catch him or herself because of limited upper extremity function and is at higher risk of injury and possible fracture after an osteotomy than a non-arthrogrypotic patient. We now discuss this specific risk with the families pre-operatively.

We have a combined approach to surgery for the upper limb in arthrogryposis utilizing multiple procedures. The timing depends on the time of presentation of the patient to our hospital. Ideally we address the elbow joint tightness with a posterior release and triceps lengthening around one year of age, to obtained passive elbow flexion to at least 90 degrees. We also perform a dorsal closing wedge osteotomy of the wrist and thenar release with first web space deepening at this time. As the child gets older, we discuss both elbow flexion transfers and distal humerus rotational osteotomies to improve hand position. When planning bilateral DHOs, we typically suggest waiting at least 3 months between surgeries to allow for osteotomy healing and to regain motion.

There are several limitations to this study. There is a broad spectrum of involvement of arthrogryposis in our patient cohort, with some patients being more involved than others in both the upper and lower extremities. The degree of involvement affects the overall clinical status, as seen with the wide range of PODCI values, but we believe we were able to detect a notable clinical change in position after DHO. Unfortunately we do not have pre-operative PODCI scores to be able to isolate specific changes in scores resulting from the DHO. Secondly, there is no validated dexterity instrument utilized to assess hand function. However, such an instrument would not be helpful in this population because of the level of involvement of their hands. Thus we felt a more subjective observational approach would be the most effective assessment tool and so we developed our own grading system to evaluate 
grasp patterns. We did not feel that it was clear enough from our videos to specifically assess grasp for each specific object (i.e. small, large, square, or cubic), thus we used the assessment of grasp to look at hand position during grasp. A third limitation was that we were not able to verify the measurements of rotation; this was a retrospective clinical study and many measurements were previously obtained. However, our therapists measure rotation based on our established convention and have had numerous years of experience.

Additionally, there were a high number of additional concurrent, and subsequent, procedures. This is a common limitation in this patient population and one that makes it somewhat more difficult to quantify subjective improvement. We did try to be focused with our evaluation, specifically looking at the resting posture of the arm and hand position for grasp, based on the hand position, the direct effect of the DHO. Lastly, we obtained an average change in resting posture of 51 degrees, but have stated that we typically rotate the distal humerus 90 degrees intra-operatively. This discrepancy is due to a change in technique over the timeframe of the study. We have become more aggressive with our degree of rotation; initially we did not utilize as much rotation and have changed our technique. Unfortunately we do not have the specific intra-operative degrees of rotation from the operative notes to quantify this. That being said, we believe that each extremity needs to be evaluated independently to position the hand facing midline and to allow the hand to come passively to the mouth.

Overall, our study has shown that distal humerus osteotomies can improve grasp patterns in patients with arthrogryposis, amyoplasia type, by redirecting the palm towards midline. We have had good success rotating the humerus approximately 90 degrees intraoperatively and utilizing a plate and screw construct for osteotomy fixation. Longer-term studies are needed to fully delineate the effect of this surgery on patients' overall clinical functional outcome.

\section{Supplementary Material}

Refer to Web version on PubMed Central for supplementary material.

\section{Acknowledgments}

This publication was supported by the Washington University Institute of Clinical and Translational Sciences grants UL1 TR000448 and TL1 TR000449 from the National Center for Advancing Translational Sciences. The content is solely the responsibility of the authors and does not necessarily represent the official views of the NIH.

\section{References}

1. Atkins RM, Bell MJ, Sharrard WJ. Pectoralis major transfer for paralysis of elbow flexion in children. J Bone Joint Surg Br. 1985; 67(4):640-644. [PubMed: 4030867]

2. Axt MW, Niethard FU, Doderlein L, Weber M. Principles of treatment of the upper extremity in arthrogryposis multiplex congenita type I. J Pediatr Orthop B. 1997; 6(3):179-185. [PubMed: 9260646]

3. Bennett JB, Hansen PE, Granberry WM, Cain TE. Surgical management of arthrogryposis in the upper extremity. J Pediatr Orthop. 1985; 5(3):281-286. [PubMed: 3998127]

4. Ezaki M. Treatment of the upper limb in the child with arthrogryposis. Hand Clin. 2000; 16(4):703711. [PubMed: 11117058]

5. Gagnon E, Fogelson N, Seyfer AE. Use of the latissimus dorsi muscle to restore elbow flexion in arthrogryposis. Plast Reconstr Surg. 2000; 106(7):1582-1585. [PubMed: 11129190] 
6. Gogola GR, Ezaki M, Oishi SN, Gharbaoui I, Bennett JB. Long head of the triceps muscle transfer for active elbow flexion in arthrogryposis. Tech Hand Up Extrem Surg. 2010; 14(2):121-124. [PubMed: 20526167]

7. Goldfarb CA, Burke MS, Strecker WB, Manske PR. The Steindler flexorplasty for the arthrogrypotic elbow. J Hand Surg Am. 2004; 29(3):462-269. [PubMed: 15140491]

8. Lahoti O, Bell MJ. Transfer of pectoralis major in arthrogryposis to restore elbow flexion: deteriorating results in the long term. J Bone Joint Surg Br. 2005; 87(6):858-860. [PubMed: 15911673]

9. Van Heest A, James MA, Lewica A, Anderson KA. Posterior elbow capsulotomy with triceps lengthening for treatment of elbow extension contracture in children with arthrogryposis. $\mathrm{J}$ Bone Joint Surg Am. 2008; 90(7):1517-1523. [PubMed: 18594101]

10. Van Heest A, Waters PM, Simmons BP. Surgical treatment of arthrogryposis of the elbow. J Hand Surg Am. 1998; 23(6):1063-1070. [PubMed: 9848560]

11. Zlotolow DA, Kozin SH. Posterior elbow release and humeral osteotomy for patients with arthrogryposis. J Hand Surg Am. 2012; 37(5):1078-1082. [PubMed: 22483178]

12. Daltroy LH, Liang MH, Fossel AH, Goldberg MJ. The POSNA pediatric musculoskeletal functional health questionnaire: report on reliability, validity, and sensitivity to change. J Pediatr Orthop. 1998; 18(5):561-571. [PubMed: 9746401]

13. Haynes RJ, Sullivan E. The Pediatric Orthopaedic Society of North America Pediatric Orthopaedic Functional Health Questionnaire: An Analysis of Normals. J Pediatr Orthop. 2001; 21(5):619-621. [PubMed: 11521030]

14. Bamshad M, Van Heest AE, Pleasure D. Arthrogryposis: A review and update. J Bone Joint Surg Amer. 2009; 91(Suppl 4):40-46.

15. Van Heest AE, Rodiguez R. Dorsal carpal wedge osteotomy in the arthrogrypotic wrist. J Hand Surg Amer. 2013; 38A:265-270.

16. Foy CA, Mills J, Wheeler L, Ezaki M, Oishi S. Long-term outcome following carpal wedge osteotomy in the arthrogrypotic patient. J Bone Joint Surg Amer. 2013; 95e150:1-6. 


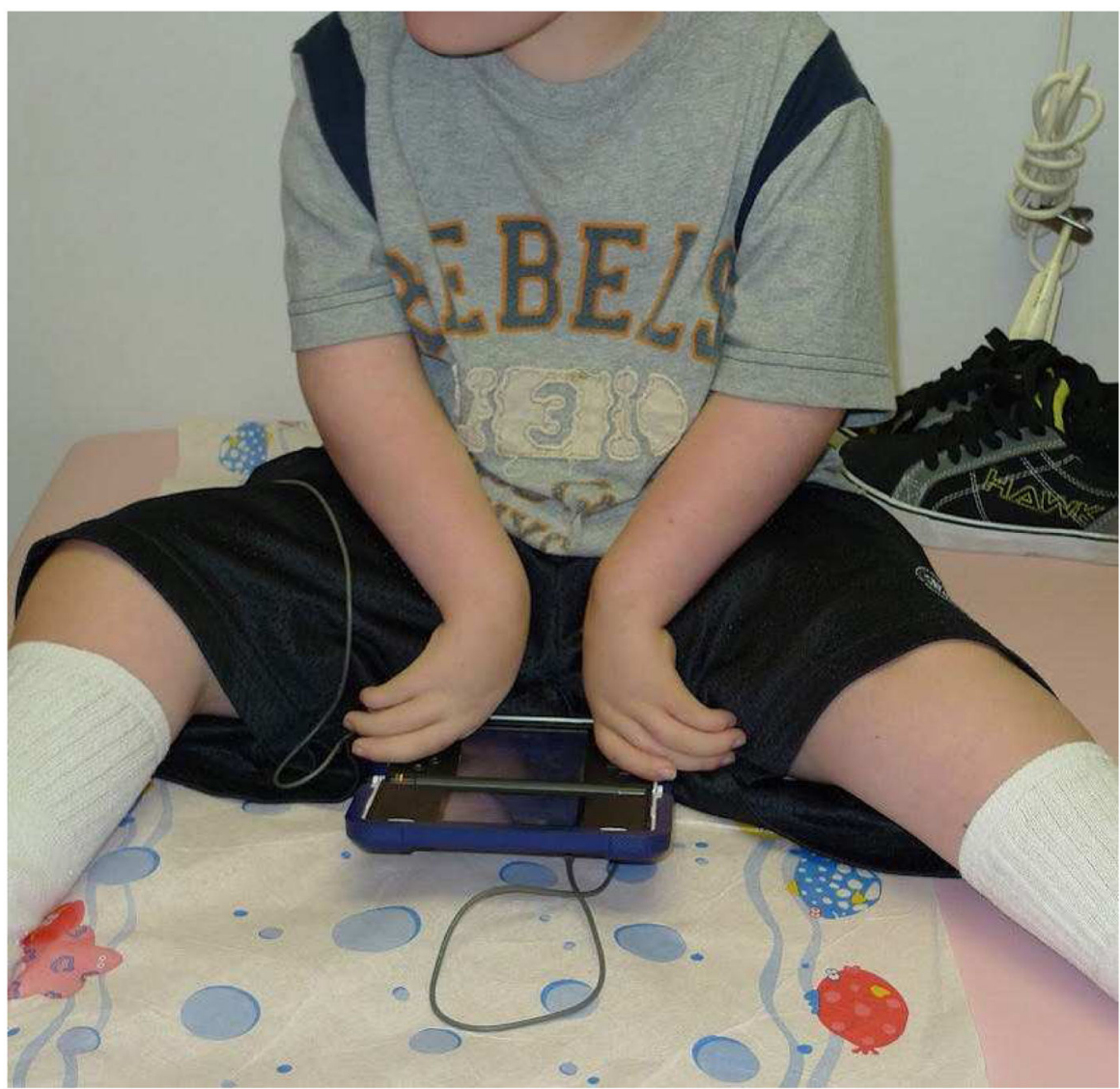

Figure 1.

A clinical photograph of the typical arthrogrypotic upper extremity posture: the shoulders remain internally rotated which typically places the hands dorsal side to dorsal side. 


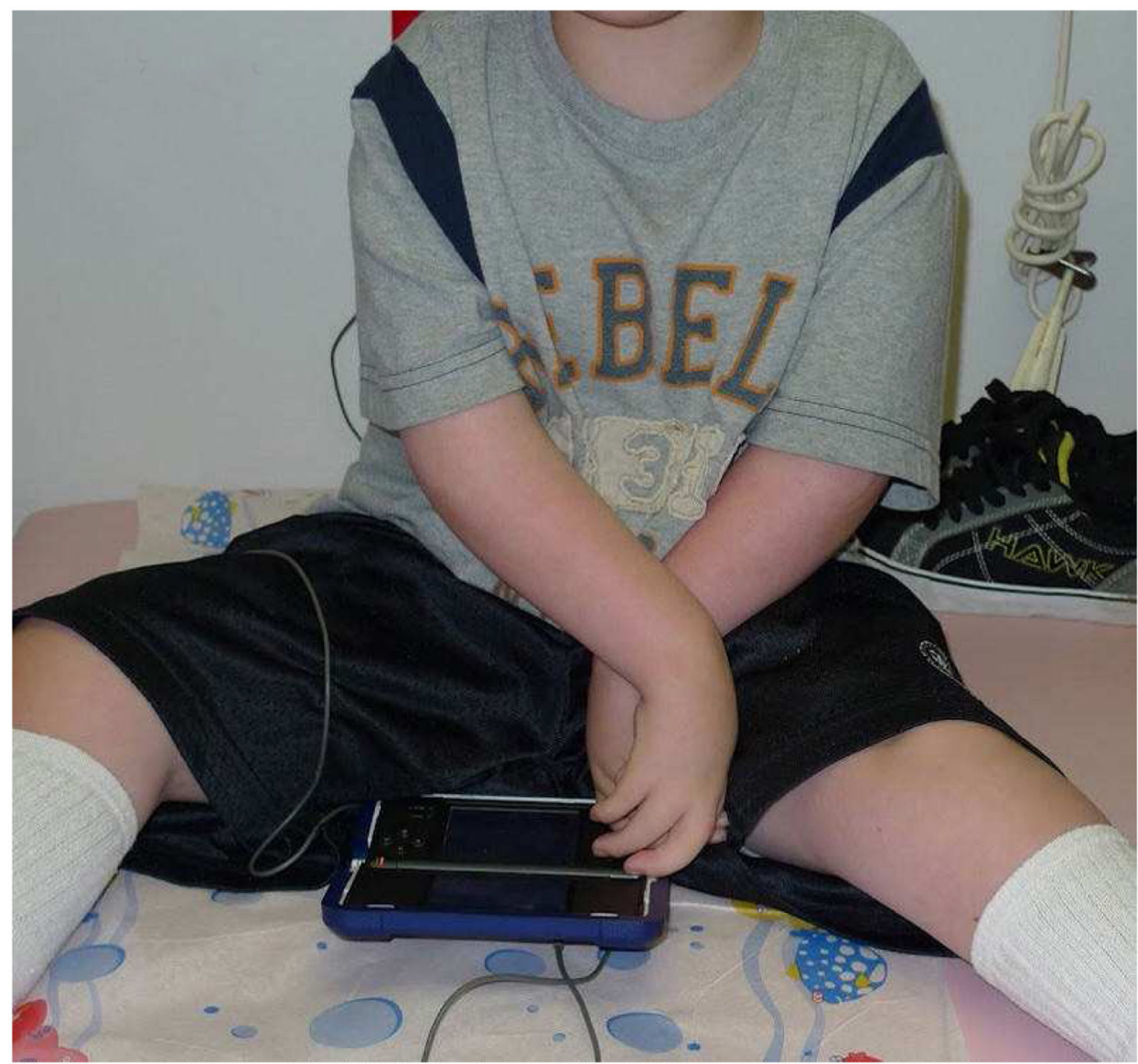

Figure 2.

A clinical photograph of the classic crossover grasp in the arthrogrypotic patient. One hand is crossed over the other to perform pal-to-palm grasp. 


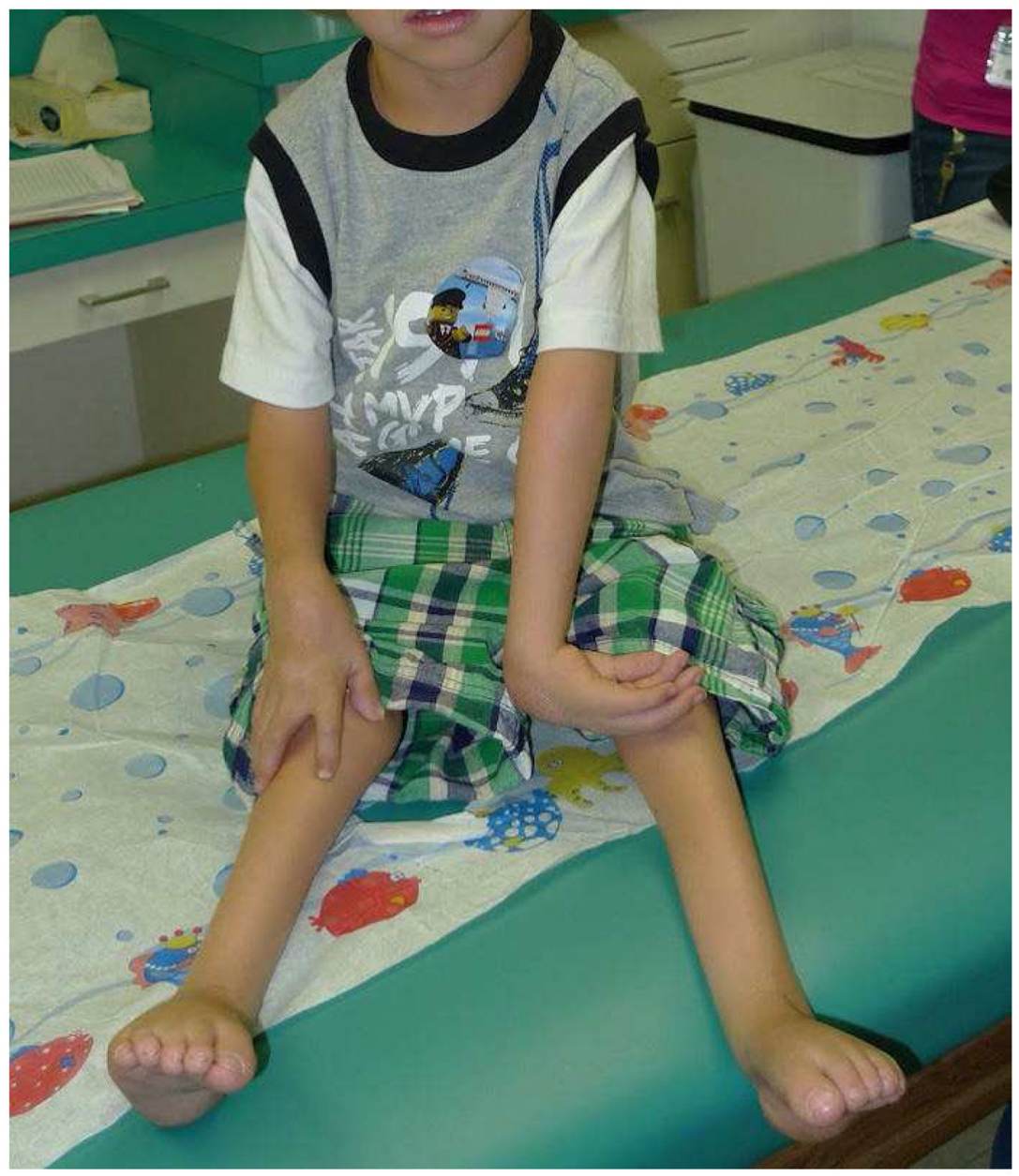

Figure 3.

Clinical image of upper extremity posture after distal humerus osteotomy on the right. Note the palm facing toward midline compared to the left that has not had DHO. 


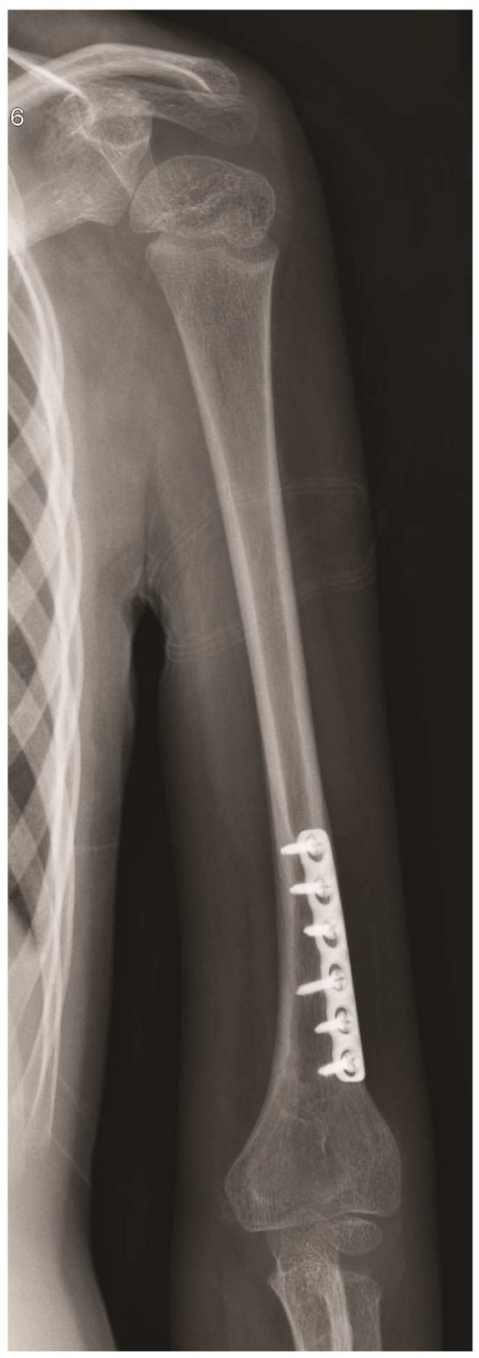




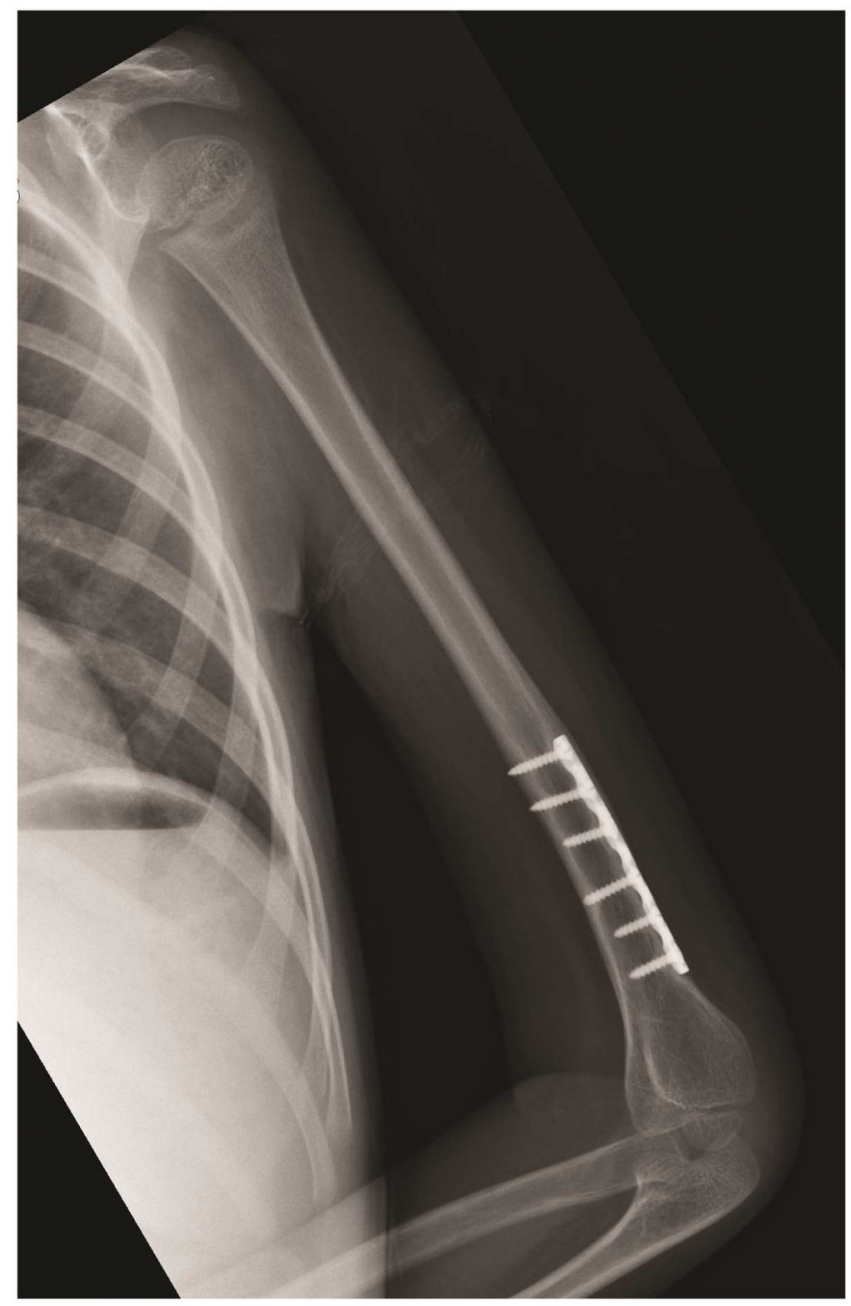

Figure 4.

Figure 4A and B. Radiographs of a healed DHO with plate and screw fixation. 


\section{Table 1}

Measurement of resting position of the upper extremity pre-operatively (pre-op) versus post-operatively (postop).

\begin{tabular}{|c|c|c|c|}
\hline Patient & Pre-op resting position & Post-op resting position & Delta \\
\hline $1 \mathrm{R}$ & $30 \mathrm{IR}$ & $15 \mathrm{IR}$ & 15 \\
\hline $1 \mathrm{~L}$ & $50 \mathrm{IR}$ & $15 \mathrm{IR}$ & 35 \\
\hline $2 \mathrm{R}$ & $48 \mathrm{IR}$ & $0 \mathrm{IR}$ & 48 \\
\hline $2 \mathrm{~L}$ & $55 \mathrm{IR}$ & $30 \mathrm{IR}$ & 25 \\
\hline $3 \mathrm{R}$ & $40 \mathrm{IR}$ & $30 \mathrm{ER}$ & 70 \\
\hline $3 \mathrm{~L}$ & $50 \mathrm{IR}$ & $15 \mathrm{ER}$ & 65 \\
\hline $4 \mathrm{R}$ & $65 \mathrm{IR}$ & $25 \mathrm{ER}$ & 40 \\
\hline $4 \mathrm{~L}$ & $65 \mathrm{IR}$ & 0 & 65 \\
\hline $5 \mathrm{R}$ & $90 \mathrm{IR}$ & $30 \mathrm{IR}$ & 60 \\
\hline $5 \mathrm{~L}$ & $90 \mathrm{IR}$ & $40 \mathrm{IR}$ & 50 \\
\hline $6 \mathrm{~L}$ & 0 & $45 \mathrm{ER}$ & 45 \\
\hline $7 \mathrm{~L}$ & $90 \mathrm{IR}$ & 0 & 90 \\
\hline $8 \mathrm{~L}$ & $50 \mathrm{IR}$ & 0 & 50 \\
\hline
\end{tabular}

$\mathrm{IR}=$ internal rotation. $\mathrm{ER}=$ external rotation. 


\section{Table 2}

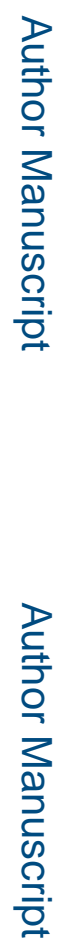

Grades of hand position.

\begin{tabular}{|c|c|c|c|}
\hline Patient & Pre-Op & Post-Op & Delta \\
\hline $\mathbf{1 R}$ & 3 & 2 & 1 \\
\hline $\mathbf{1 L}$ & 2 & 1 & 1 \\
\hline $\mathbf{2 R}$ & 2 & 2 & 0 \\
\hline $\mathbf{2} \mathbf{R}$ & 2 & 1 & 1 \\
\hline $\mathbf{3 R}$ & 3 & 1 & 2 \\
\hline $\mathbf{3 L}$ & 3 & 1 & 2 \\
\hline $\mathbf{4 R}$ & 3 & 1 & 2 \\
\hline $\mathbf{4 L}$ & 3 & 2 & 1 \\
\hline $\mathbf{5 R}$ & 3 & 2 & 1 \\
\hline $\mathbf{5 L}$ & 3 & 2 & 1 \\
\hline $\mathbf{6 L}$ & 3 & 1 & 2 \\
\hline $\mathbf{7 L}$ & 2 & 1 & 1 \\
\hline $\mathbf{8 L}$ & 3 & 1 & 2 \\
\hline
\end{tabular}

Grade 1 = palms facing midline, Grade 2 = palm facing posterior, Grade 3 = palm facing away from the body 


\section{Table 3}

\section{Parent responses to DHO surgery.}

\begin{tabular}{|l|}
\hline Quotes from parents response: \\
\hline 1. Improved function "a great deal" \\
\hline 2. "Its just easier now" \\
\hline 3. “...it seems like he has more function with his arms turned" \\
\hline 4. "It's a lot easier for her to do things --like writing." \\
\hline 5. "She can hold her pencil properly without any struggle." \\
\hline 6. She is so happy! \\
\hline
\end{tabular}

